\title{
Input/Output Linearization: A Nonlinear Analog of Placing Poles at Process Zeros
}

This paper deals with SISO nonlinear processes and their control with nonlinear static state feedback. The Byrnes-Isidori concept of nonlinear zeros is first reviewed and illustrated with two chemical engineering examples. This concept is then used to interpret input/ output linearizing state feedback as a nonlinear analog of placing poles at the process zeros. This interpretation leads to closed-loop internal stability conditions for nonlinear processes under input/output linearizing state feedback.

\author{
Costas Kravaris \\ Department of Chemical Engineering \\ University of Michigan \\ Ann Arbor, Ml 48109
}

\section{Introduction}

A key element of modern linear control theory is that a controller must generate an approximate inverse of the process transfer function (Garcia and Morari, 1982). Alternatively stated, a controller must be synthesized in such a way that closed-loop poles are located at the process zeros (provided they are all in the left half-plane). The wide acceptance of this philosophy is due to the resulting optimality properties in terms of the integral square error (ISE) criterion. The purpose of this work is to extend the concept of placing poles at the process zeros to nonlinear systems and demonstrate its optimality characteristics.

A question that immediately arises is, "What do we mean by zeros of a nonlinear system?" Recent work by Byrnes and Isidori (1985) has developed a very meaningful concept of nonlinear zeros using methods and results from differential geometry. In our work we will use this concept to develop for the first time a concept of placing poles at the process zeros to nonlinear systems. We will show that the class of input/output linearizing state feedback laws (Kravaris and Chung, 1987) places poles at the process zeros in a nonlinear process. This will lead to natural internal stability conditions for input/output linearizing state feedback.

The significance of the concept of nonlinear zeros is not limited to process control; it has potential applicability in the area of process design. One major specification when designing a process and its operating conditions is to avoid right-half-plane zeros, which give rise to inverse response characteristics and limit the quality of control. We now have the appropriate tools for achieving this goal in nonlinear processes.

The next two sections provide a precise statement of wellknown linear results which will be subsequently extended to nonlinear systems. Subsequent sections provide the necessary background from differential geometry, a review of the Byrnes-
Isidori concept of nonlinear zeros, and illustrations with two chemical engineering examples. The final two sections interpret input/output linearizing state feedback as a nonlinear analog of placing poles at the process zeros, and provide internal stability conditions for input/output linearizing state feedback and discuss its ISE optimality characteristics.

\section{ISE-Optimal Pole Placement for Linear Systems: Letov's Rule}

Consider a linear system

$$
\begin{aligned}
& \dot{x}=A x+b u \\
& y=c x
\end{aligned}
$$

where $u \in R, x \in R^{n}, y \in R$, and $A, b, c$ are matrices of appropriate dimensions. The transfer function of the above system is given by

$$
\frac{y(s)}{u(s)}=c(s I-A)^{-1} b=\frac{c \operatorname{Adj}(s I-A) b}{\operatorname{det}(s I-A)}
$$

When such a system is subject to static state feedback

$$
u=v-k x
$$

its transfer function becomes

$$
\frac{y(s)}{v(s)}=\frac{c \operatorname{Adj}(s I-A) b}{\operatorname{det}(s I-A+b k)}
$$

State feedback alters only the poles of the transfer function. The well-known pole placement theorem (Kailath, 1980; Chen, 
1971) states that given an arbitrary set of pole locations, there is always a unique $k$ that places the poles at the given locations (assuming of course that the system is controllable).

A natural question to ask is what is the optimal $k$ that minimizes a given performance criterion? Equivalently, what are the closed-loop pole locations that minimize the given performance criterion? The well-known theorem of Letov (1960) states that for ISE optimality in a controllable and observable system, poles must be placed:

- At the left-half-plane zeros of the process

- At the mirror images of the right-half-plane zeros with respect to the imaginary axis

- The remaining poles far left in the complex plane

The ISE criterion will become minimal in the limit as the far left poles tend to negative infinity.

\section{ISE-Optimal State Feedback Law for Minimum-Phase Systems}

Consider again the linear system of Eqs. 1, whose transfer function is given by Eq. 2. A formal Taylor series expansion of $(s I-A)^{-1}$ shows that the system's transfer function is completely determined by the quantities $c b, c A b, c A^{2} b, \ldots, c A^{k-1} b$, ... These are called Markov parameters of the system. It turns out (Kailath, 1980; Chen, 1971) that the relative order $r$ of the system-that is, the difference between the degrees of $\operatorname{det}(s I-A)$ and $c A d j(s I-A) b$-is equal to the smallest integer $r$ for which

$$
c A^{r-1} b \neq 0
$$

A basic property of static state feedback is that it preserves relative order. In other words, if the open-loop system has relative order $r$, then the closed-loop system has relative order $r$ as well.

Proposition 1. Consider the linear system of Eqs. 1 and assume that its relative order is $r$. Then the state feedback

$$
u=v-\frac{1}{c A^{r-1} b}\left(\sum_{k=0}^{r} \beta_{k} c A^{k}\right) x
$$

places the poles at the roots of the $(n-r)$ th degree polynomial $c A d j(s I-A) b$ and at the roots of the $r$ th degree polynomial $\Sigma_{k-0}^{\prime} \beta_{k} s^{k}$. The resulting closed-loop transfer function is of the form

$$
\frac{y(s)}{v(s)}=\frac{\text { constant }}{\beta_{r} s^{r}+\beta_{r-1} s^{r-1}+\cdots+\beta_{1} s+\beta_{0}}
$$

The state feedback, Eq. 5, cancels all the zeros of the process by placing poles at them. It is clear that the closed-loop system will be internally stable if and only if all the zeros of Eqs. 1 are in the left half-plane, that is, if and only if the process is minimum phase.

Moreover, for a minimum-phase process, Letov's rule establishes that the closed-loop system will be ISE-optimal in the limit as the roots of $\Sigma_{k-0}^{r} \beta_{k} s^{k}$ tend to negative infinity.

Finally, it is interesting to note that the closed-loop system, Eq. 6, is of order $r$. This is the minimum possible order under a state feedback of the form of Eq. 3.

\section{Useful Concepts and Results from Differential Geometry \\ Integral curves of a vector field}

Given a vector field $g$ and a point $x_{0}=\left(x_{10}, x_{20}, \ldots, x_{n 0}\right)$ on an $(n-1)$-dimensional surface $S$, let $\phi_{i}\left(\theta ; x_{0}\right), i=1, \ldots, n$ be the solution of

$$
\begin{aligned}
& \frac{d \phi_{i}}{d \theta}=g_{i}\left(\phi_{1}, \ldots, \phi_{n}\right) \\
& \phi_{i}(0)=x_{i 0}
\end{aligned}
$$

For each $\theta \in R,\left(\phi_{1}, \ldots, \phi_{n}\right)$ defines a point in $R^{n}$. Thus $\left[\phi_{1}\left(\theta ; x_{0}\right), \ldots, \phi_{n}\left(\theta ; x_{0}\right)\right]$ defines a curve in $R^{n}$ starting from the point $x_{0}$; this curve is called the integral curve of $g$ passing from the point $x_{0}$.

Integral curves have the following basic property: At each point in $R^{n}, g$ is always tangent to its integral curve passing from that point.

An ( $n-1)$-dimensional surface $S$ is called characteristic to a vector field $g$ if $g$ is tangent to the surface at each of its points. Equivalently, if for every $x_{0} \in S$, the corresponding integral curve $\phi\left(\theta ; x_{0}\right)$ of $g$ lies in $S$. An $(n-1)$-dimensional surface $S$ is called noncharacteristic to a vector field $g$ if $g$ is nontangent to the surface at each of its points. Equivalently, if for every $x_{0} \in$ $S$, the intersection of the integral curve $\phi\left(\theta ; x_{0}\right)$ of $g$ with $S$ is exactly the point $x_{0}$. (The terminology "characteristic" and "noncharacteristic" originates from the theory of partial differential equations; see Courant and Hilbert, 1962, p. 73.)

\section{Invertible coordinate transformations}

The scalar fields $\Xi_{1}(x), \Xi_{2}(x), \ldots, \Xi_{m}(x)$ are called linearly independent if their gradients $d \Xi_{1}, d \Xi_{2}, \ldots, d \Xi_{m}$ are linearly independent vectors of $R^{n}$.

Consequently, a transformation

$$
\begin{gathered}
\zeta_{1}=\Xi_{1}\left(x_{1}, x_{2}, \ldots, x_{n}\right) \\
\zeta_{2}=\Xi_{2}\left(x_{1}, x_{2}, \ldots, x_{n}\right) \\
\vdots \\
\zeta_{n}=\Xi_{n}\left(x_{1}, x_{2}, \ldots, x_{n}\right)
\end{gathered}
$$

is invertible if and only if the scalar fields $\Xi_{1}(x), \Xi_{2}(x), \ldots$, $\Xi_{n}(x)$ are linearly independent.

An invertible transformation $x \in R^{n} \rightarrow \zeta=\Xi(x) \in R^{n}$ defines a curvilinear coordinate system. The new coordinates are $\zeta_{1}, \zeta_{2}, \ldots, \zeta_{n}$; the basis vectors of the new coordinate system are

$$
\begin{gathered}
\overline{e_{1}}=\text { first column of }\left(\frac{\partial \Xi}{\partial x}\right)^{-1} \\
\overline{e_{2}}=\text { second column of }\left(\frac{\partial \Xi}{\partial x}\right)^{-1} \\
\vdots \\
\overline{e_{n}}=n \text {th column of }\left(\frac{\partial \Xi}{\partial x}\right)^{-1}
\end{gathered}
$$


It is important to mention here that when a vector field is transformed in curvilinear coordinates, its new components will be along $\overline{e_{1}}, \overline{e_{2}}, \ldots, \overline{e_{n}}$ rather than

$$
\left[\begin{array}{c}
1 \\
0 \\
\vdots \\
0
\end{array}\right],\left[\begin{array}{c}
0 \\
1 \\
\vdots \\
0
\end{array}\right] \cdots\left[\begin{array}{c}
0 \\
0 \\
\vdots \\
1
\end{array}\right]
$$

\section{"Straightening out" a vector field}

A classical problem of differential geometry is that of straightening out a vector field. More precisely, given a vector field $g$, find a curvilinear coordinate system

$$
\left(\zeta_{1}, \zeta_{2}, \ldots, \zeta_{n}\right)=\left[\Xi_{1}(x), \Xi_{2}(x), \ldots, \Xi_{n}(x)\right]
$$

such that $g=\bar{e}_{n}$, the $n$th basis vector of the curvilinear coordinate system. This means that all components of $g$ along $\zeta_{1}$, $\zeta_{2}, \ldots, \zeta_{n-1}$ will be zero and the component of $g$ along $\zeta_{n}$ will equal 1 . Consequently, the integral curves of $g$ in the $\zeta$-coordinate system will be straight lines; it is in this sense that $g$ is "straightened out."

Clearly, the requirement $g=\bar{e}_{n}$ is equivalent to

$$
\frac{\partial \Xi}{\partial x} g=\left[\begin{array}{c}
0 \\
0 \\
\vdots \\
1
\end{array}\right]
$$

Thus, one can equivalently pose the problem as follows: Given a vector field $g$, find $n$ linearly independent scalar fields $\Xi_{1}(x)$, $\Xi_{2}(x), \ldots, \Xi_{n}(x)$ such that

$$
\begin{gathered}
\left\langle d \Xi_{1}, g\right\rangle=0 \\
\vdots \\
\left\langle d \Xi_{n-1}, g\right\rangle=0 \\
\left\langle d \Xi_{n}, g\right\rangle=1
\end{gathered}
$$

In the following we outline a simple algorithm for generating a curvilinear coordinate system that straightens out a given vector field g. (For more details, see Hicks, 1965, p. 124, or other differential geometry texts that contain a constructive proof of the theorem of Frobenius.)

Let $S$ be a surface described by an equation of the form $x_{n}=$ $\Upsilon\left(x_{1}, \ldots, x_{n-1}\right)$ which:

1. Passes through the origin, that is,

$$
\Upsilon(0, \ldots, 0)=0
$$

2. Is noncharacteristic around the origin, that is,

$$
\sum_{i=1}^{n-1} \frac{\partial \Upsilon}{\partial x_{i}} g_{i}\left(x_{1}, \ldots, x_{n-1}, \Upsilon\right) \neq g_{n}\left(x_{1}, \ldots, x_{n-1}, \Upsilon\right)
$$

Further, let

$$
\begin{gathered}
x_{1}=\phi_{1}\left(\theta ; \zeta_{1}, \ldots, \zeta_{n-1}\right) \\
\vdots \\
x_{n-1}=\phi_{n-1}\left(\theta ; \zeta_{1}, \ldots, \zeta_{n-1}\right) \\
x_{n}=\phi_{n}\left(\theta ; \zeta_{1}, \ldots, \zeta_{n-1}\right)
\end{gathered}
$$

be the integral curves of $g$ originating from the point of $S$. $\left[\zeta_{1}, \zeta_{2}, \ldots, \zeta_{n-1}, \Upsilon\left(\zeta_{1}, \zeta_{2}, \ldots, \zeta_{n-1}\right)\right]$

In other words, Eqs. 14 are the solution of:

$$
\begin{array}{cl}
\frac{d x_{1}}{d \theta}=g_{1}\left(x_{1}, \ldots, x_{n}\right), & x_{1}(0)=\zeta_{1} \\
& \vdots \\
\frac{d x_{n-1}}{d \theta}=g_{n-1}\left(x_{1}, \ldots, x_{n}\right), \quad x_{n-1}(0)=\zeta_{n-1} \\
\frac{d x_{n}}{d \theta}=g_{n}\left(x_{1}, \ldots, x_{n}\right), \quad x_{n}(0)=\Upsilon\left(\zeta_{1}, \zeta_{2}, \ldots, \zeta_{n-1}\right)
\end{array}
$$

Then the inverse function

$$
\begin{aligned}
& \zeta_{1}=\Xi_{1}\left(x_{1}, \ldots, x_{n}\right) \vdots \\
& \zeta_{n-1}=\Xi_{n-1}\left(x_{1}, \ldots, x_{n}\right) \\
& \theta=\Xi_{n}\left(x_{1}, \ldots, x_{n}\right)
\end{aligned}
$$

defined from Eqs. 14 is the necessary coordinate transformation.

Remark 1. By construction, the coordinate transformation of Eqs. 16 maps the origin to the origin. If it is desired to obtain a coordinate transformation that maps the point $x^{*}$ to the origin, then one must compute the integral curve of $g$ originating from

$$
\left[x_{1}^{*}+\zeta_{1}, x_{2}^{*}+\zeta_{2}, \ldots, x_{n-1}^{*}+\zeta_{n-1}, x_{n}^{*}-\Upsilon\left(\zeta_{1}, \zeta_{2}, \ldots, \zeta_{n-1}\right)\right]
$$

and invert

Remark 2. Since the noncharacteristic surface $S$ as well as the choice of the $n$th coordinate $x_{n}$ is completely arbitrary, the coordinate transformation of Eqs. 16 is inherently nonunique.

Remark 3. If $g_{n}\left(x_{1}, \ldots, x_{n}\right) \neq 0$, then the surface $x_{n}=0$ is noncharacteristic and passes through the origin. This is often the most convenient choice for $S$. (If $g_{n}=0$ we can always rotate the indices of $x_{1}, \ldots, x_{n}$ to make $g_{n} \neq 0$ ).

\section{Zero Dynamics: A Generalization of the Concept of Zeros to Nonlinear Systems}

\section{Zero dynamics of a linear system}

Consider for the moment a linear system of the form of Eqs. 1 whose relative order is $r$ and $b \neq 0$. It is easy to see that the row vectors $c, c A, \ldots, c A^{r-1}$ are linearly independent. Thus, one can 
always rotate the indices of the state variables $x_{1}, \ldots, x_{n}$ so that:

1. $(r-1)$ linearly independent columns of the matrix

$$
\left[\begin{array}{c}
c \\
c A \\
\vdots \\
c A^{r-2}
\end{array}\right]
$$

are in the $(n-r+1)$ th, $(n-r+2)$ th, $\ldots(n-1)$ th places.

2. $b_{n} \neq 0$.

Then, it is not difficult to verify that the transformation

$$
\begin{aligned}
\zeta_{1}= & x_{1}-\frac{b_{1}}{b_{n}} x_{n} \\
& \vdots \\
\zeta_{n-r} & =x_{n-r}-\frac{b_{n-r}}{b_{n}} x_{n} \\
\zeta_{n-r+1} & =c x \\
& \vdots \\
\zeta_{n-1} & =c A^{r-2} x \\
\zeta_{n} & =c A^{r-1} x
\end{aligned}
$$

is invertible and transforms Eqs. 1 into a system of the form

$$
\begin{aligned}
{\left[\begin{array}{c}
\dot{\zeta}_{1} \\
\vdots \\
\dot{\zeta}_{n-r}
\end{array}\right]=\tilde{A}\left[\begin{array}{c}
\zeta_{1} \\
\vdots \\
\zeta_{n-r}
\end{array}\right]+\Gamma\left[\begin{array}{c}
\zeta_{n-r+1} \\
\vdots \\
\zeta_{n}
\end{array}\right] } \\
\dot{\zeta}_{n-r+1}=\zeta_{n-r+2} \\
\vdots \\
\dot{\zeta}_{n-1}=\zeta_{n} \\
\dot{\zeta}_{n}=\tilde{a}_{n}\left[\begin{array}{c}
\zeta_{1} \\
\vdots \\
\zeta_{n-r}
\end{array}\right]+\gamma_{n}\left[\begin{array}{c}
\zeta_{n-r+1} \\
\vdots \\
\zeta_{n}
\end{array}\right]+\beta u \\
y=\zeta_{n-r+1}
\end{aligned}
$$

where $\tilde{A}, \Gamma, \tilde{a}_{n}, \gamma_{n}$ are $(n-r) \times(n-r),(n-r) \times r, 1 \times$ $(n-r), 1 \times r$ matrices, respectively, and $\beta$ is a nonzero scalar.

The dynamic system of Eqs. 18 is just a different realization of the transfer function, Eq. 2. What is interesting about the realization is that the eigenvalues of the $(n-r) \times(n-r)$ matrix $\tilde{A}$ are exactly the zeros of Eq. 2 . Indeed, the transfer function of Eqs. 18, $y(s) / u(s)$, is easily found to be:

$$
\frac{\beta \operatorname{det}(s I-\tilde{A})}{s^{r} \operatorname{det}(s I-\tilde{A})-\left[\gamma_{n} \operatorname{det}(s I-\tilde{A})+\tilde{a}_{n} \operatorname{Adj}(s I-\tilde{A}) \Gamma\right]\left[\begin{array}{c}
1 \\
s \\
\vdots \\
s^{-1}
\end{array}\right]}
$$

Definition 1. Consider the dynamic system of Eqs. 1, which is transformed into Eqs. 18 with an appropriate similarity transformation. Then the dynamic system

$$
\left[\begin{array}{c}
\dot{z}_{1} \\
\vdots \\
\dot{z}_{n-r}
\end{array}\right]=\tilde{A}\left[\begin{array}{c}
z_{1} \\
\vdots \\
\vdots \\
z_{n-r}
\end{array}\right]+\Gamma\left[\begin{array}{c}
U_{1} \\
\vdots \\
\vdots \\
U_{r}
\end{array}\right]
$$

is called the zero dynamics of Eqs. 1 .

The dynamic system

$$
\left[\begin{array}{c}
\dot{z}_{1} \\
\vdots \\
\dot{z}_{n-r}
\end{array}\right]=\tilde{A}\left[\begin{array}{c}
z_{1} \\
\vdots \\
z_{n-r}
\end{array}\right]
$$

is called the unforced zero dynamics of Eqs. 1.

Another interesting byproduct of the canonical form, Eqs. 18, is obtaining a minimal order realization of the process inverse. It is not difficult to verify that

$$
\begin{gathered}
{\left[\begin{array}{c}
\dot{z}_{1} \\
\vdots \\
\dot{z}_{n-r}
\end{array}\right]=\tilde{A}\left[\begin{array}{c}
z_{1} \\
\vdots \\
z_{n-r}
\end{array}\right]+\Gamma\left[\begin{array}{c}
y \\
\vdots \\
\frac{d^{r-1} y}{d t^{r-1}}
\end{array}\right]} \\
\left.u=\frac{1}{\beta}\left(\begin{array}{c}
z_{1} \\
\vdots \\
\vdots \\
z_{n-r}
\end{array}\right]-\gamma_{n}\left[\begin{array}{c}
y \\
\vdots \\
\frac{d^{r-1} y}{d t^{r-1}}
\end{array}\right]+\frac{d^{r} y}{d t^{r}}\right)
\end{gathered}
$$

is a realization of

$$
\frac{u(s)}{y(s)}=\frac{\operatorname{det}(s I-A)}{c \operatorname{Adj}(s I-A) b}
$$

\section{Zero dynamics of a nonlinear system}

All the results presented in the previous subsection generalize to nonlinear systems. This generalization was due to Byrnes and Isidori (1985) and its importance is far beyond the minimal order realization of the process inverse: it allows generalizing 
the concept of zeros to nonlinear systems. The main results and definitions are outlined below.

Consider a nonlinear system of the form

$$
\begin{aligned}
& \dot{x}=f(x)+g(x) u \\
& y=h(x)
\end{aligned}
$$

where $u \in R, y \in R, x \in R^{n}, f(x)$, and $g(x)$ are vector fields on $R^{n}$ and $h(x)$ is a scalar field on $R^{n}$.

Definition 2. (Hirschorn, 1979) The relative order of the nonlinear system of Eqs. 22 is the smallest integer $r$ for which

$$
\left\langle d h, \operatorname{ad}_{f}^{r-1}(g)\right\rangle \neq 0
$$

A comparison with the linear case shows that the above concept of relative order is a natural extension. Indeed,

$$
(-1)^{k-1}\left\langle d h, a d_{f}^{k-1}(g)\right\rangle
$$

becomes $c A^{k-1} b$ when $f(x)=A x, g(x)=b, h(x)=c x$.

Proposition 2. Consider a nonlinear system of the form of Eqs. 22 whose relative order is $r$. Then the scalar fields $h$, $L_{f}(h), \ldots, L_{f}^{r-2}(h), L_{f}^{r-1}(h)$ are linearly independent.

An immediate consequence of the proposition is that the $(r-1) \times n$ matrix

$$
\left[\begin{array}{ccc}
\frac{\partial h}{\partial x_{1}} & \cdots & \frac{\partial h}{\partial x_{n}} \\
\frac{\partial L_{f}(h)}{\partial x_{1}} & \cdots & \frac{\partial L_{f}(h)}{\partial x_{n}} \\
\vdots & \vdots \\
\frac{\partial L_{f}^{r-2}(h)}{\partial x_{1}} & \cdots & \frac{\partial L_{f}^{r-2}(h)}{\partial x_{n}}
\end{array}\right]
$$

has rank $(r-1)$. Therefore, we can always rotate the indices of the state variables $x_{1}, \ldots, x_{n}$ so that

$$
\operatorname{det}\left[\begin{array}{ccc}
\frac{\partial h}{\partial x_{n-r+1}} & \cdots & \frac{\partial h}{\partial x_{n-1}} \\
\vdots & & \vdots \\
\frac{\partial L_{f}^{r-2}(h)}{\partial x_{n-r+1}} & \cdots & \frac{\partial L_{f}^{r-2}(h)}{\partial x_{n-1}}
\end{array}\right] \neq 0
$$

Also, with rotation of indices we can assure that

$$
g_{n} \neq 0
$$

Theorem 1. (Byrnes-Isidori, 1985) Consider a system of the form of Eqs. 22 whose relative order is $r$. Assume that the indices of $x_{i}$ have been rotated so that Eqs. 23 and 24 are satisfied and let $\left[t_{1}(x), \ldots, t_{n}(x)\right]$ be a curvilinear coordinate system that straightens out the vector field $g$. Then the transformation

$$
\zeta=T(x)=\left[\begin{array}{c}
t_{1}(x) \\
\vdots \\
t_{n-r}(x) \\
h(x) \\
L_{f}(h)(x) \\
\vdots \\
L_{f}^{r-2}(h)(x) \\
L_{f}^{r-1}(h)(x)
\end{array}\right]
$$

is invertible and transforms Eqs. 22 into

$$
\begin{aligned}
\dot{\zeta}_{1} & =F_{1}(\zeta) \\
& \vdots \\
\dot{\zeta}_{n-r} & =F_{n-r}(\zeta) \\
\dot{\zeta}_{n-r+1} & =\zeta_{n-r+2} \\
& \vdots \\
\dot{\zeta}_{n-1} & =\zeta_{n} \\
\zeta_{n} & =\Phi(\zeta)+G(\zeta) u \\
y & =\zeta_{n-r+1}
\end{aligned}
$$

where

$$
\begin{gathered}
F_{i}=\left\langle d t_{i}, f\right\rangle, i=1, \ldots, n-r \\
\Phi=L_{f}^{r}(h) \\
G=(-1)^{r-1}\left\langle d h, a d_{f}^{r-1}(g)\right\rangle
\end{gathered}
$$

The transformation Eq. 25 is a direct generalization of the transformation Eqs. 17 obtained for a linear system. Also, the transformed system of Eqs. 26 is a direct generalization of Eqs. 18 , in which $F_{i}, \Phi$, and $G$ are linear functions.

The result about the process inverse generalizes as well; it is a corollary of the previous theorem:

Corollary. Consider a nonlinear system of the form of Eqs. 22 whose relative order is $r$ and assume that it has been transformed into Eqs. 26 through an invertible transformation of the form of Eq. 25. Then the dynamic system

$$
\begin{aligned}
\dot{z}_{1} & =F_{1}\left(z_{1}, \ldots, z_{n-r}, y, \frac{d y}{d t}, \ldots, \frac{d^{r-1} y}{d t^{r-1}}\right) \\
& : \\
\dot{z}_{n-r}= & F_{n-r}\left(z_{1}, \ldots, z_{n-r}, y, \frac{d y}{d t}, \ldots, \frac{d^{r-1} y}{d t^{r-1}}\right) \\
u & =\frac{\frac{d^{\prime} y}{d t^{r}}-\Phi\left(z_{1}, \ldots, z_{n-r}, y, \frac{d y}{d t}, \ldots, \frac{d^{r-1} y}{d t^{r-1}}\right)}{G\left(z_{1}, \ldots, z_{n-r}, y, \frac{d y}{d t}, \ldots, \frac{d^{r-1} y}{d t^{r-1}}\right)}
\end{aligned}
$$

is a minimal order realization of the inverse of the nonlinear system of Eqs. 22. 
Clearly, the stability properties of the inverse are completely determined by the stability properties of the dynamic system

$$
\begin{aligned}
\dot{z}_{1} & =F_{1}\left(z_{1}, \ldots, z_{n-r}, U_{1}, \ldots, U_{r}\right) \\
& \vdots \\
& : \\
\dot{z}_{n-r} & =F_{n-r}\left(z_{1}, \ldots, z_{n-r}, U_{1}, \ldots, U_{r}\right)
\end{aligned}
$$

In particular, the unforced inverse $\{y(t)=[d y / d t(t)]=\ldots=$ $d^{r-1} y / d t^{r-1}=0$ \} will be asymptotically stable if and only if

$$
\begin{aligned}
\dot{z}_{1} & =F_{1}\left(z_{1}, \ldots, z_{n-r}, 0, \ldots, 0\right) \\
& \vdots \\
& : \\
\dot{z}_{n-r} & =F_{n-r}\left(z_{1} \ldots, z_{n-r}, 0, \ldots, 0\right)
\end{aligned}
$$

is asymptotically stable.

In linear systems, the zeros of a system are the poles of the inverse. Therefore the zeros can be thought of as a set of numbers that determine the dynamics of the inverse. With this in mind, it is natural to try to extend the concept of zeros to nonlinear systems by considering the dynamics of a minimal order realization of the process inverse. This leads to the following definition.

Definition 3. Consider a nonlinear system of the form of Eqs. 22 whose relative order is $r$ and an invertible transformation $\zeta=$ $T(x)$ of the form of Eq. 25 that transforms Eqs. 22 into Eqs. 26. Assume that with appropriate translation of axes the origin is an equilibrium point of Eqs. 26. The (forced) zero dynamics of Eqs. 22 is the $(n-r)$-order dynamic system

$$
\begin{aligned}
\dot{z}_{1} & =F_{1}\left(z_{1}, \ldots, z_{n-r}, U_{1}, \ldots, U_{r}\right) \\
& \vdots \\
& : \\
\dot{z}_{n-r} & =F_{n-r}\left(z_{1}, \ldots, z_{n-r}, U_{1}, \ldots, U_{r}\right)
\end{aligned}
$$

In particular, the unforced zero dynamics is the $(n-r)$-order unforced dynamic system

$$
\begin{aligned}
\dot{z}_{1} & =F_{1}\left(z_{1}, \ldots, z_{n-r}, 0, \ldots, 0\right) \\
& \vdots \\
& \vdots \\
\dot{z}_{n-r} & =F_{n-r}\left(z_{1}, \ldots, z_{n-r}, 0, \ldots, 0\right)
\end{aligned}
$$

The next step is to try to define a nonlinear analog of a minimum phase system, that is, of all zeros in the left half-plane. This cannot be done in a unique way; it will depend on the concept of stability used for the zero dynamics, Eqs. 31. Byrnes and Isidori (1985) define minimum-phase nonlinear systems as those that have asymptotically stable unforced zero dynamics, Eqs. 32. Depending on the context, more or less stringent stability requirements will be necessary. In the final section we will use the following stability concept for zero dynamics:

Definition 4. Under the assumptions of definition 3, we will say that the nonlinear system of Eqs. 22 has stable zero dynamics if for any set of initial conditions $z_{1}(0), \ldots, z_{n-r}(0)$ and any exponentially decaying $U_{1}, \ldots, U_{r}$,

$$
\lim _{t \rightarrow \infty} z_{i}(t)=0 \quad i=1, \ldots, n-r
$$

\section{Examples of Zero Dynamics Example 1: CSTR}

Consider a continuously stirred tank reactor (CSTR) in which an isothermal, liquid-phase, multicomponent chemical reaction is being carried out. The chemical reaction system is $A \rightleftharpoons B \rightarrow C$, with the rates of reaction given by:

$$
\begin{aligned}
& r_{1}=k_{1} C_{A}-k_{2} C_{B}^{2} \\
& r_{2}=k_{3} C_{B}^{2}
\end{aligned}
$$

It is desired to control the concentration of species $C$ by manipulating the molar feed rate of $B, N_{B F}$, as shown in Figure 1.

The modeling equations for this system are

$$
\begin{aligned}
\frac{d C_{A}}{d t} & =\frac{F}{V}\left(C_{A F}-C_{A}\right)-k_{1} C_{A}+k_{2} C_{B}^{2} \\
\frac{d C_{B}}{d t} & =-\frac{F}{V} C_{B}+k_{1} C_{A}-k_{2} C_{B}^{2}-k_{3} C_{B}^{2}+u \\
\frac{d C_{C}}{d t} & =-\frac{F}{V} C_{C}+k_{3} C_{B}^{2} \\
y & =C_{C}
\end{aligned}
$$

where

$$
u=\frac{N_{B F}}{V}
$$

This system has relative order $r=2$. Indeed

$$
\begin{aligned}
h & =C_{C}, & \langle d h, g\rangle & =0 \\
L_{f}(h) & =-\frac{F}{V} C_{C}+k_{3} C_{B}^{2}, & \left\langle d L_{f}(h), g\right\rangle & \neq 0
\end{aligned}
$$

Notice that

$$
g=\left[\begin{array}{l}
0 \\
1 \\
0
\end{array}\right],
$$

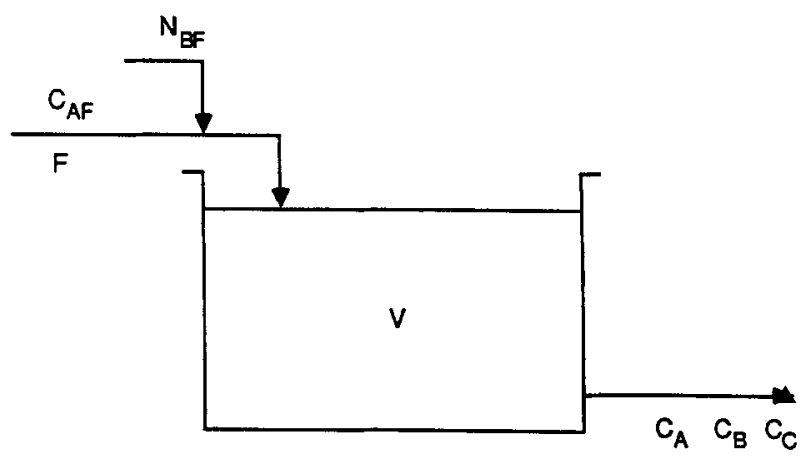

Figure 1. System for example 1. 
so it is already straight. To make it

$$
\left[\begin{array}{l}
0 \\
0 \\
1
\end{array}\right]
$$

as required by our convention, all we need to do is rotate the states

$$
\begin{aligned}
& x_{1}=C_{A} \\
& x_{2}=C_{C} \\
& x_{3}=C_{B}
\end{aligned}
$$

With this rotation

$$
\begin{aligned}
& \frac{\partial h}{\partial x_{\mathrm{n}-r+1}} \equiv \frac{\partial h}{\partial x_{2}}=1 \neq 0 \\
& g_{3}=1 \neq 0
\end{aligned}
$$

Thus Eqs. 23 and 24 are satisfied. Consequently, theorem 1 tells us that the transformation

$$
T(x)=\left[\begin{array}{c}
C_{A} \\
C_{C} \\
-\frac{F}{V} C_{C}+k_{3} C_{B}^{2}
\end{array}\right]
$$

will transform Eqs. 33 to the Byrnes-Isidori canonical form, Eqs. 26. Since we usually want the canonical form to have the origin as an equilibrium point, we must do an appropriate axis translation, that is, define

$$
\begin{aligned}
& \zeta_{1}=C_{A}-C_{A s} \\
& \zeta_{2}=C_{C}-C_{C s} \\
& \zeta_{3}=-\frac{F}{V}\left(C_{C}-C_{C s}\right)+k_{3}\left(C_{B}^{2}-C_{B s}^{2}\right)
\end{aligned}
$$

where $\left(u_{s}, C_{A s}, C_{B s}, C_{C_{s}}\right)$ denotes the operating steady state.

In the coordinate system of Eqs. 34, Eqs. 33 become:

$$
\begin{aligned}
\dot{\zeta}_{1}= & -\left(\frac{F}{V}+k_{1}\right) \zeta_{1}+\frac{k_{2}}{k_{3}}\left(\frac{F}{V} \zeta_{2}+\zeta_{3}\right) \\
\dot{\zeta}_{2}= & \zeta_{3} \\
\dot{\zeta}_{3}= & -\frac{F}{V}\left[2 k_{3} C_{B s}^{2}+\frac{F}{V} \zeta_{2}+3 \zeta_{3}\right] \\
& +2 k_{3}\left[C_{B s}^{2}+\frac{1}{k_{3}}\left(\frac{F}{V} \zeta_{2}+\zeta_{3}\right)\right]^{0.5} \\
& \cdot\left[\frac{F}{V} C_{B s}+k_{1} \zeta_{1}-\left(1+\frac{k_{2}}{k_{3}}\right)\left(\frac{F}{V} \zeta_{2}+\zeta_{3}\right)\right] \\
& +2 k_{3}\left[C_{B s}^{2}+\frac{1}{k_{3}}\left(\frac{F}{V} \zeta_{2}+\zeta_{3}\right)\right]^{0.5}\left(u-u_{s}\right) \\
y= & \zeta_{2}
\end{aligned}
$$

According to definition 3, the (forced) zero dynamics of Eqs. 33 is simply the first equation of Eqs. 35 with inputs $U_{1}=\zeta_{2}$ and $U_{2}=\zeta_{3}$, that is,

$$
\dot{z}=-\left(\frac{F}{V}+k_{1}\right) z+\frac{k_{2}}{k_{3}}\left(\frac{F}{V} U_{1}+U_{2}\right)
$$

Clearly, Eqs. 36 is stable (in any sense) and therefore the system of Eqs. 33 has stable zero dynamics.

\section{Example 2: Continuous antibiotic fermentor}

An important class of bioengineering processes comprises antibiotic fermentations. The control objective is to maintain the cell mass at a given level (which will maximize antibiotic production) by manipulating the dilution rate of the substrate.

Mass balances for the cells and the substrate give

$$
\begin{aligned}
& \frac{d X}{d t}=\mu(S, X) X-D X \\
& \frac{d S}{d t}=-\sigma(S, X) X+D\left(S_{F}-S\right)
\end{aligned}
$$

where $\mu(S, X)$ and $\sigma(S, X)$ are known functions of $S$ and $X$ that depend on the particular fermentation system.

Defining

$$
\begin{aligned}
& u=D=\text { manipulated input } \\
& y=X=\text { controlled output }
\end{aligned}
$$

we can put the model, Eqs. 37, into standard form as follows:

$$
\frac{d}{d t}\left[\begin{array}{l}
X \\
S
\end{array}\right]=\left[\begin{array}{c}
\mu(S, X) X \\
-\sigma(S, X) X
\end{array}\right]+\left[\begin{array}{c}
-X \\
S_{F}-S
\end{array}\right] u
$$

Let us compute the zero dynamics of the nonlinear system of Eqs. 38. Denoting by $\left(D_{s}, X_{s}, S_{s}\right)$ the operating steady state of the fermentor, we first need to compute the integral curve of the vector field

$$
g=\left[\begin{array}{c}
-X \\
S_{F}-S
\end{array}\right]
$$

that originates from the point $S=S_{s}+\zeta$ of the noncharacteristic surface $X=X_{s}$. This is computed by solving

$$
\begin{array}{lr}
\frac{d X}{d \theta}=-X, & X(0)=X_{s} \\
\frac{d S}{d \theta}=S_{F}-S, & S(0)=S_{s}+\zeta
\end{array}
$$

We easily find

$$
\begin{aligned}
& X=X_{s} e^{-\theta} \\
& S=\left(S_{s}-S_{F}+\zeta\right) e^{-\theta}+S_{F}
\end{aligned}
$$

The inverse function defined as the solution of the above equa- 
tion for $\zeta$ and $\theta$ will provide a coordinate transformation that straightens out the vector field

$$
g=\left[\begin{array}{c}
-X \\
S_{F}-S
\end{array}\right]
$$

and maps the point $\left(X_{s}, S_{s}\right)$ to the origin. We find

$$
\begin{aligned}
& \zeta=\left(S-S_{F}\right) \frac{X_{s}}{X}-\left(S_{s}-S_{F}\right) \\
& \theta=\ln \left(\frac{X_{s}}{X}\right)
\end{aligned}
$$

Remark 4. It is easy to verify that the basis vectors of the above coordinate transformation are

$$
\overline{e_{\zeta}}=\left[\begin{array}{c}
0 \\
\frac{X}{X}
\end{array}\right], \overline{e_{\theta}}=\left[\begin{array}{c}
-X \\
S_{F}-S
\end{array}\right] \text {, }
$$

so it indeed straightens out

$$
g=\left[\begin{array}{c}
-X \\
S_{F}-S
\end{array}\right]
$$

The next step is to compute the relative order of our system. We easily find $r=1$. Thus, Theorem 1 tells us that the transformation

$$
\begin{aligned}
& \zeta=\left(S-S_{F}\right) \frac{X_{s}}{X}-\left(S_{s}-S_{F}\right) \\
& \zeta=X-X_{s}
\end{aligned}
$$

will transform Eqs. 38 into the canonical form, Eqs. 26. Indeed, a straightforward calculation gives:

$$
\begin{aligned}
\frac{d \zeta}{d t} & =-\tilde{\sigma}\left(\zeta, \zeta^{\prime}\right) X_{s}-\tilde{\mu}\left(\zeta, \zeta^{\prime}\right)\left(S_{s}-S_{F}+\zeta\right) \\
\frac{d \zeta^{\prime}}{d t} & =\tilde{\mu}\left(\zeta, \zeta^{\prime}\right)\left(X_{s}+\zeta^{\prime}\right)-u\left(X_{s}+\zeta^{\prime}\right) \\
y & =\zeta^{\prime}
\end{aligned}
$$

where

$$
\begin{aligned}
& \tilde{\mu}\left(\zeta, \zeta^{\prime}\right)=\mu\left[\left(\left(S_{s}-S_{F}+\zeta\right)\left(1+\frac{\zeta^{\prime}}{X_{s}}\right)+S_{F}, X_{s}+\zeta^{\prime}\right)\right] \\
& \tilde{\sigma}\left(\zeta, \zeta^{\prime}\right)=\sigma\left[\left(\left(S_{s}-S_{F}+\zeta\right)\left(1+\frac{\zeta^{\prime}}{X_{s}}\right)+S_{F}, X_{s}+\zeta^{\prime}\right)\right]
\end{aligned}
$$

According to definition 3, the (forced) zero dynamics of Eqs. 38 is simply the first equation of the transformed system, Eqs. 43, with input $U=\zeta^{\prime}$, that is,

$$
\frac{d z}{d t}=-\tilde{\sigma}(z, U) X_{s}-\tilde{\mu}(z, U)\left(S_{s}-S_{F}+z\right)
$$

In particular, the unforced zero dynamics $(U=0)$ is

$$
\frac{d z}{d t}=-\sigma\left(S_{s}+z, X_{s}\right) X_{s}-\mu\left(S_{s}+z, X_{s}\right)\left(S_{s}-S_{F}+z\right)
$$

Clearly, the stability characteristics of the zero dynamics will depend upon

- The functions $\mu(S, X)$ and $\sigma(S, X)$

- The operating steady state $X_{s}$

For a specific fermentation system ( $\mu$ and $\sigma$ given), we will in general obtain conditional stability results depending on the operating steady state $X_{s}$.

\section{Interpretation of Input/Output Linearizing State Feedback as Canceling the Zero Dynamics of a Process}

Consider again a nonlinear system of the form of Eqs. 22:

$$
\begin{aligned}
& \dot{x}=f(x)+g(x) u \\
& y=h(x)
\end{aligned}
$$

The problem of input/output linearization was posed by Kravaris and Chung (1987) as that of finding a static state feedback $u=\Psi(x, v)$ so that the resulting closed-loop system has linear input/output behavior of minimal order. In particular, it is requested that the closed-loop system obey a linear differential equation of the form

$$
\sum_{k} \beta_{k} \frac{d^{k} y}{d t^{k}}=v
$$

where the $\beta_{k}$ 's are adjustable parameters, selected a priori by the designer. The solution is summarized in the following theorem:

Theorem 2. (Kravaris and Chung, 1987) Consider a nonlinear system of the form of Eqs. 22 whose relative order is $r$. Then

a. The minimal order of the closed-loop system is equal to $r$.

b. The necessary state feedback that makes the closed-loop input/output system linear and of minimal order is given by

$$
u=\frac{v-\sum_{k=0}^{r} \beta_{k} L_{f}^{k}(h)}{(-1)^{r-1} \beta_{r}\left\langle d h, a d_{f}^{r-1}(g)\right\rangle}
$$

where $\beta_{k}$ are arbitrarily selected numbers. 
The input/output behavior of the closed-loop system is then governed by

$$
\sum_{k=0}^{r} \beta_{k} \frac{d^{k} y}{d t^{k}}=v
$$

Consider now an invertible transformation

$$
\zeta=T(x)=\left[\begin{array}{c}
t_{1}(x) \\
\vdots \\
t_{n-r}(x) \\
h(x) \\
L_{f}(h)(x) \\
\vdots \\
L_{f}^{r-2}(h)(x) \\
L_{f}^{r-1}(h)(x)
\end{array}\right]
$$

that transforms Eqs. 22 into the Byrnes-Isidori canonical form:

$$
\begin{aligned}
\dot{\zeta}_{1} & =F_{1}\left(\zeta_{1}, \ldots, \zeta_{n-r}, \zeta_{n-r+1}, \ldots, \zeta_{n-1}, \zeta_{n}\right) \\
& \vdots \\
\dot{\zeta}_{n-r} & =F_{n-r}\left(\zeta_{1}, \ldots, \zeta_{n-r}, \zeta_{n-r+1}, \ldots, \zeta_{n-1}, \zeta_{n}\right) \\
\dot{\zeta}_{n-r+1} & =\zeta_{n-r+2} \\
& \vdots \\
& : \\
\dot{\zeta}_{n-1} & =\zeta_{n} \\
\dot{\zeta}_{n} & =L_{f}^{r}(h)+(-1)^{r-1}\left\langle d h, a d_{f}^{r-1}(g)\right\rangle u \\
y & =\zeta_{n-r+1}
\end{aligned}
$$

The first $n-r$ equations, when viewed as a dynamic system with inputs $\zeta_{n-r+1}, \ldots, \zeta_{n-1}, \zeta_{n}$ represent the zero dynamics. The output $y=\zeta_{n-r+1}$ is affected by the zero dynamics through the righthand side of the $n$th equation. Therefore, in order to cancel the zero dynamics, we need a state feedback that makes

$$
L_{f}^{r}(h)+(-1)^{r-1}\left\langle d h, a d_{f}^{r-1}(g)\right\rangle u
$$

a function of $\zeta_{n-r+1}, \ldots, \zeta_{n}$ and $v$ only, independent of $\zeta_{1}, \ldots$, $\zeta_{n-r}$

In particular, we may request it to be a linear function

$$
\begin{aligned}
L_{f}^{r}(h)+(-1)^{r-1}\left\langle d h, a d_{f}^{r-1}(g)\right\rangle u \\
=\frac{1}{\beta_{r}}\left(v-\beta_{0} \zeta_{n-r+1}-\cdots-\beta_{r-1} \zeta_{n}\right)
\end{aligned}
$$

This leads to the closed-loop system

$$
\begin{aligned}
\dot{\zeta}_{1} & =F_{1}\left(\zeta_{1}, \ldots, \zeta_{n-r}, \zeta_{n-r+1}, \ldots, \zeta_{n-1}, \zeta_{n}\right) \\
& \vdots \\
\dot{\zeta}_{n-r} & =F_{n-r}\left(\zeta_{1}, \ldots, \zeta_{n-r}, \zeta_{n-r+1}, \ldots, \zeta_{n-1}, \zeta_{n}\right) \\
\dot{\zeta}_{n-r+1} & =\zeta_{n-r+2} \\
& \vdots \\
& : \\
\dot{\zeta}_{n-1} & =\zeta_{n} \\
\dot{\zeta}_{n} & =-\frac{\beta_{0}}{\beta_{r}} \zeta_{n-r+1}-\cdots-\frac{\beta_{r-1}}{\beta_{r}} \zeta_{n}+\frac{1}{\beta_{r}} v \\
y & =\zeta_{n-r+1}
\end{aligned}
$$

whose output is completely unaffected by the first $n-r$ equations.

Notice that Eq. 49 can be equivalently written as

$$
u=\frac{\left(v-\beta_{0} \zeta_{n-r+1}-\cdots-\beta_{r-1} \zeta_{n}\right)-\beta_{r} L_{f}^{r}(h)}{(-1)^{r-1} \beta_{r}\left\langle d h, a d_{f}^{r-1}(g)\right\rangle}
$$

or in terms of the original variables

$$
u=\frac{v-\beta_{0} h-\cdots-\beta_{r-1} L_{f}^{r-1}(h)-\beta_{r} L_{f}^{r}(h)}{(-1)^{r-1} \beta_{r}\left\langle d h, a d_{f}^{r-1}(g)\right\rangle}
$$

But this is identical to Eq. 46 !

It is interesting to point out here the remarkable analogies between input/output linearizing state feedback and the linear state feedback considered earlier. When $f(x)=A x, g(x)=b$, and $h(x)=c x$, Eq. 46 becomes

$$
u=\frac{v}{\beta_{r} c A^{r-1} b}-\frac{1}{\beta_{r} c A^{r-1} b}\left(\sum_{k=0}^{r} \beta_{k} c A^{k}\right) x
$$

which is identical to Eq. 5 under appropriate rescaling of $u$ and $v$. This state feedback places poles at the zeros of Eq. 2 or equivalently cancels the zero dynamics of Eqs. 1.

\section{Internal Stability and ISE Optimality Properties of Input/Output Linearizing State Feedback}

In this section we present internal stability and ISE optimality results for the input/output linearizing state feedback, which are in complete analogy to the earlier linear results for minimum-phase systems.

Theorem 3. Consider the nonlinear system of Eqs. 22 with the state feedback of Eq. 46 and assume that $\beta_{0}, \ldots, \beta_{r}$ have been chosen so that the roots of the polynomial $\beta_{r} s+\ldots+\beta_{1} s+\beta_{0}$ are in the open left half-plane. The closed-loop system will be internally stable if the zero dynamics of the open-loop system (Eqs. 22) are stable in the sense of definition 4.

Proof. From Eqs. 50 we have that the unforced closed-loop 
system is governed by

$$
\begin{aligned}
\dot{\zeta}_{1} & =F_{1}\left(\zeta_{1}, \ldots, \zeta_{n-r}, \zeta_{n-r+1}, \ldots, \zeta_{n-1}, \zeta_{n}\right) \\
& \vdots \\
\dot{\zeta}_{n-r} & =F_{n-r}\left(\zeta_{1}, \ldots, \zeta_{n-r}, \zeta_{n-r+1}, \ldots, \zeta_{n-1}, \zeta_{n}\right) \\
\dot{\zeta}_{n-r+1} & =\zeta_{n-r+2} \\
& \vdots \\
& : \\
\dot{\zeta}_{n-1} & =\zeta_{n} \\
\dot{\zeta}_{n} & =-\frac{\beta_{0}}{\beta_{r}} \zeta_{n-r+1}-\ldots-\frac{\beta_{r-1}}{\beta_{r}} \zeta_{n}
\end{aligned}
$$

Since the roots of the polynomial $\beta_{r} s+\cdots+\beta_{1} s+\beta_{0}$ are in the open left half-plane, any deviations of $\zeta_{n-r+1}(0), \ldots$, $\zeta_{n-1}(0), \zeta_{n}(0)$ from the equilibrium will generate exponentially decaying $\zeta_{n-r+1}(t), \ldots, \zeta_{n-1}(t), \zeta_{n}(t)$; these will be the inputs of the zero dynamics of definition 3 . The result follows immediately from definition 4.

The most important conclusion from this theorem is that for $\beta_{r} s^{r}+\cdots+\beta_{1} s+\beta_{0}$ having all roots in the open left halfplane, internal stability depends on the intrinsic characteristics of the open-loop system; these have been associated with stable zero dynamics in the sense of definition 4 . If an open-loop system has stable zero dynamics, then the closed-loop system will be internally stable no matter what the values of $\beta_{k}$ are (as long as they are in the left half-plane).

Theorem 4. Consider the nonlinear system of Eqs. 22, whose relative order is $r$ and assume that it has stable zero dynamics in the sense of definition 4 . Then the state feedback

$$
u=\frac{v-h-\beta_{1} L_{f}(h)-\cdots-\beta_{r} L_{f}^{r}(h)}{(-1)^{r-1} \beta_{r}\left\langle d h, a d_{f}^{r-1}(g)\right\rangle}
$$

provides ISE-optimal response to step changes in $v$ in the limit as the roots of the polynomial $\beta_{r} s^{r}+\cdots+\beta_{1} s+1$ tend to negative infinity.

The proof is straightforward given the expression for the closed-loop response, Eq. 47, and standard linear results.

\section{Notation}

$C_{A}=$ concentration of species $A$ in reactor

$C_{B}=$ concentration of species $B$ in reactor

$C_{C}=$ concentration of species $C$ in reactor

$D=$ dilution rate of substrate

$\bar{e}_{i}=$ basis of curvilinear cordinate system

$F=$ volumetric feed rate

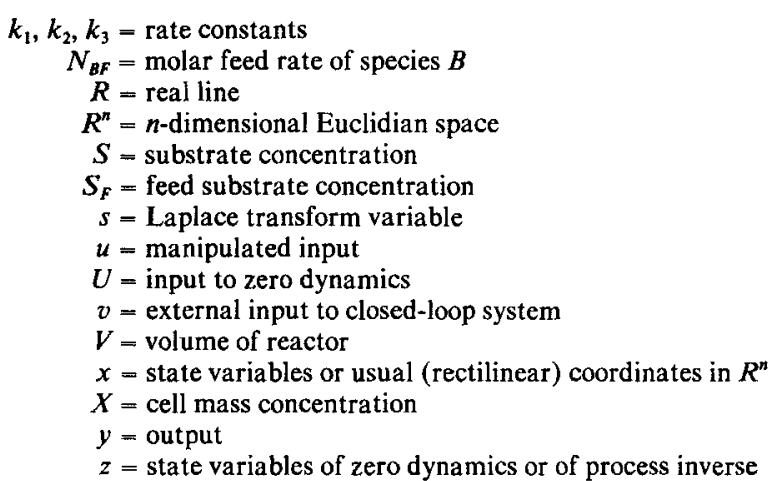

\section{Greek letters}

$\zeta=$ transformed state variables or curvilinear coordinates $\mu(S, X)=$ specific growth, a function of $S$ and $X$ $\sigma(S, X)=$ specific substrate utilization rate, a function of $S$ and $X$

\section{Mathematical notation}

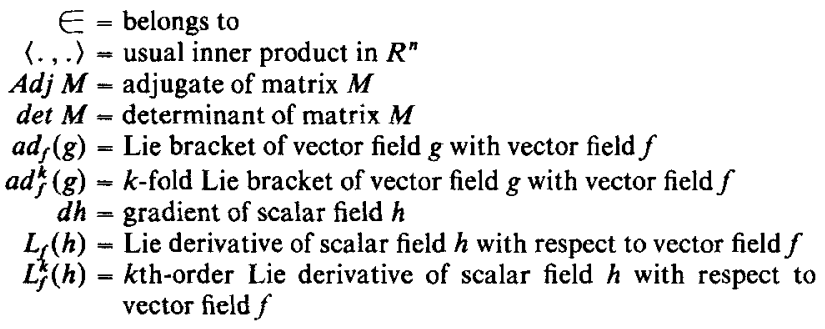

\section{Literature Cited}

Byrnes, C. I., and A. Isidori, "Global Feedback Stabilization of Nonlinear Systems," Proc. 24th IEEE CDC, Ft. Lauderdale, 1031 (1985).

Chen, C. T., Linear Systems Theory and Design, Holt, Rinehart, Winston, New York (1971).

Courant, R., and D. Hilbert, Methods of Mathematical Physics, II, Interscience, New York (1962).

Hicks, N.J., Notes on Differential Geometry, Van Nostrand, Princeton (1965).

Garcia, C. E., and M. Morari, "Internal Model Control. 1: A Unifying Review and Some New Results," Ind. Eng. Chem. Process Des. Dev., 21, 308 (1982).

Hirschorn, R. M., "Invertibility of Nonlinear Control Systems," SIAM J. Control Optim., 17, 289 (1979).

Kailath, T,, Linear Systems, Prentice-Hall, Englewood Cliffs, NJ (1980).

Kravaris, C., and C. B. Chung, "Nonlinear State Feedback Synthesis by Global Input/Output Linerization," AIChE J., 33, 592 (1987).

Letov, A. M., "Analytical Controller Design. I, II," Autom. Remote Control, 21, 303 (1960)

Manuscript received Jan. 5, 1988, and revision received June 17, 1988. 